\title{
Long term prediction of nonlinear viscoelastic creep behaviour of elastomers: extended Schapery model
}

\author{
Hatem Gacem $^{1,2, a}$, Yvon Chevalier ${ }^{1}$, Jean-Luc Dion ${ }^{1}$, Mohamed Soula $^{2}$ \\ AND BRAHIM REZGUI ${ }^{2}$ \\ 1 Laboratoire d'ingénierie des systèmes mécaniques et des matériaux (LISMMA), Institut Supérieur de Mécanique \\ de Paris (SUPMECA), 3 rue Fernand Hainaut, 93407 Saint-Ouen Cedex, Paris, France \\ ${ }^{2}$ Unité de recherche génie des matériaux (URGM), École nationale d'ingénieurs de Tunis (ENIT), BP 37, Le Belvédère, \\ Tunis, Tunisie
}

Received 18 January 2008, Accepted 4 November 2008

\begin{abstract}
Herein we develop a creep test method for characterizing nonlinear viscoelastic behaviour of an elastomer through weak deformation. This approach is based on an expanded Schapery model. Short term creep tests at various stress levels were performed upon specimens composed of a synthetic elastomer. A method to predict the long term creep behaviour using short term creep tests is presented. Experimental results and theoretical predictions are compared.
\end{abstract}

Key words: Creep tests / nonlinear visco-elasticity / Schapery model / time-stress equivalence

Résumé - Prévision à longue durée du comportement viscoélastique non-linéaire en fluage des élastomères : extension du modèle de Schapery. Dans ce travail, nous développons une méthode de caractérisation en fluage du comportement viscoélastique non-linéaire d'un élastomère en petites déformations. Cette approche est basée sur une généralisation du modèle de Schapery. Des essais de fluage à court terme et à différents niveaux de contraintes ont été menés sur des éprouvettes en élastomère synthétique. Une méthode de prévision du comportement en fluage à long terme par le biais d'essais de fluage à court terme est présentée. Une comparaison entre les résultats expérimentaux et les prévisions à partir du modèle théorique est discutée.

Mots clés : Essais de fluage / visco-élastcité non-linéaire / modèle de Schapery / équivalence contrainte-temps

\section{Introduction}

Elastomers have great industrial applications. Their physical properties encourage their use in various applications for which alternative materials do not exist. Elastomers are characterized by complex, nonlinear and dissipative behaviour. Some of them present high damping which depends more or less on frequency and temperature and offer excellent frequency properties for vibration and acoustic isolation. Composite material with elastomer matrix are used since a lot of decades because of their special properties (low mass density, high failure modulus and high damping factor), the use of such composite

${ }^{a}$ Corresponding author: gacem_hatem@yahoo.fr material began at early 1900's when captive balloons were designed and built in Germany and UK:

- Researches in domestic fields focus on pliable composites materials used as membranes in the area of moving homes and protection of environment: moving dam used to control water level and to prevent flooding

- Researches in transports, the fields deals with stiff elastomer composite materials: tires, silent blocks, steel/elastomer laminates absorber in brake systems.

Elastomer behaviour is generally considered as viscohyperelastic, quasi-incompressible and non-linear. Use of elastomer materials becomes increasingly frequent in aeronautic and automotive industries. Generally, these materials are employed for the security of dynamic 


\section{Nomenclature}

\begin{tabular}{|ll|}
\hline$a_{\mathrm{T}}:$ time-temperature multiplying factor & \\
$a_{\sigma}:$ time-stress multiplying factor & $\mathrm{MPa}$ \\
$D_{\mathrm{i}}:$ stress step & \\
$E:$ error on constant shift factor & \\
$g_{1}, g_{2}:$ stress dependent factors & $\mathrm{MPa}^{-1}$ \\
$J:$ creep compliance & $\mathrm{s}$ \\
$t:$ actual time & $\mathrm{s}$ \\
$\tau:$ elapsed time & \\
$\alpha_{\sigma}:$ generalized time-stress multiplying factor & \\
$\lambda\left(\sigma_{\mathrm{n}}\right):$ constant horizontal shift factor (at short time) & \\
$\xi\left(\sigma_{\mathrm{n}}\right):$ time dependent horizontal shift factor & \\
$\psi(\mathrm{t}):$ reduced time & $\mathrm{s}$ \\
$\psi_{0}(t):$ actual reduced time & $\mathrm{s}$ \\
$\psi_{\mathrm{e}}(t):$ elapsed reduced time & $\mathrm{s}$ \\
$\sigma:$ cauchy stress tensor & $\mathrm{MPa}$ \\
$\varepsilon:$ linear strain & \\
$\Phi:$ reduced creep strain & $\mathrm{MPa}^{-1}$ \\
$X:$ derivative of $X$ per time & \\
\hline
\end{tabular}

systems. Such uses require detailed knowledge of constitutive equations, in particular the behaviour displayed when functioning in extreme conditions of temperature or strain inducing various physical phenomena.

To model elastomer behaviour, finite deformations, nonlinearity, dissipative processes and temperature effects must simultaneously be taken into account. Various theories have been developed to perfect theoretical and numerical databases in order to describe the global and local behaviour of complex elastomer systems. Based on experimental characterization, elastomer models may be subdivided into two categories. The first is phenomenological, drawing on global behaviour while the second category is based on micro structural properties.

Elastomer behaviour can be considered as a superimposition of hyper-elasticity, damage and viscoelasticity. Thus, the behaviour is visco-hyperelastic.

Various theories have been developed to model the nonlinear viscoelastic behaviour of elastomers. A lot of approaches extend the classic idea of linear visco-elasticity by the introduction of hereditary integrals. Volterra's series are a very general approach when looking at this point of view $[1,2]$, for example, developed a constitutive visco-hyperelastic law as a function of parameters describing both equilibrium and instantaneous responses. Narrowing to single hereditary integral is more convenient when developing non-linear viscoelasticity laws because the non-linearity is expressed as non-linear creep or relaxation functions. This approach is very close to linear viscoelasticity and can be easily generalized to large deformations, see O'Dowd and Knauss [3], Lianis [4], Hausler and Sayir [5] Gacem [6]. The most famous approach in this field is Schapery model examined in Section 2.1 Schapery $[7,8]$. Schapery model is a simple construction generalizing line viscoelastic creep behaviour on the assumption of small strains. The model lays in two points:

- Stress expands make contracts artificially time by the way of shift factor.
- Stress and hereditary integral are weighted respectively by two functions which depend on stress and equal to 1 for small stress (linear behaviour).

The success of this non-linear viscoelastic Schapery model is that it suits really:

- to various loadings, behaviours, fractures, Schapery [12], damages, Schapery [13], Hinterhoelzl and Schapery [14];

- to various materials and applications, Schapery [15$18]$.

The present study seeks to present an approach for nonlinear viscoelastic characterization of elastomers using measurements from short term creep tests. This approach is based on an extended Schapery model. Afterwards, we shall develop a method for deriving the dependent long term behaviour from short term creep measurements. The method is based on developing a convenient shift factor expression which involves time dependence, Gacem [6]. Experiments are conducted on synthetic carbon black filled elastomer. This material is used in manufacturing traditional multilayer plate incorporating metallic and visco-elastic materials for machine suspension. In addition, this composite structure is used in brake system for vibro-acoustic isolation. The visco-elastic material is worked as a damping layer. Finally, we compare the theoretical predictions and experimental results.

\section{Nonlinear viscoelastic modelling}

Majority of polymers and rubber materials, at ambient temperature can be modeled by linear viscoeslastic laws in the field of small strains. Some of them, such as carbon black filled elastomers, show a non linear behaviour. When undergoing finite deformations, viscoelastic materials display non linear behaviours generated both 
by large deformations and the nature of materials. Structural response of viscoelastic material is affected by temperature mainly through thermal expansion and change in the relaxation and retardation time. When $\Delta T$ is the increase of temperature above some reference value $T$, reduced time $\psi$ is defined by the differential equation

$$
\mathrm{d} \psi=\frac{\mathrm{d} t}{a_{T}}
$$

$a_{T}=a_{T}[T(t)]$ is often called time-temperature multiplying factor, Ferry [19], Morland and Lee [20], Lee and Roberts [21] which depends of actual time $t$, geometric and behavioural nonlinearity. Various theories have been developed for modeling the nonlinear viscoelastic behaviour of different elastomers. Schapery's model, close to linear viscoelasticity Schapery [22] and rheological model, is commonly used for the purpose of modeling nonlinear viscoelastic behaviour in the case of polymers and elastomers.

\subsection{Schapery model}

A nonlinear thermo-viscoelastic model has been developed by Schapery. This model is expressed by a simple integral form and is based on the thermodynamics of irreversible process theory introducing internal variables which take into account the history of the material from some initial point in time [23].

In his model Schapery uses the Onsager relations (symmetry of creep and relaxation functions for anisotropic materials) based on thermodynamic approach [24-26] and generalizes Lee's suggestions [20,21]. The physical basis for this description derives from the observation that stress induced dilatation effects the mobility of molecular chains through changing the free volume of material. The empirical concept described by Lee is finally enlarged by Knauss and Emri [27, 28] by including, beside temperature, the effect of stress and solvent concentration induced volume dilatation. The time dependence (viscosity) is modified by a time multiplying factor a which depends on the temperature $T$, mechanical dilation $\theta$ (inducing stress $\sigma$ ) and solvent concentration $c$,

$$
a=a[T, \sigma(\theta), c]
$$

Doolittle [29] expressed this factor in terms of free volume by an empirical logarithmic law taking up again by Knauss et al. [27]. The Schapery model, in situation of temperature $T$ and solvent concentration constant $c$, may be expressed in deformation response form as follows:

$$
\begin{aligned}
\varepsilon(t)= & \left\{g_{1}\left[\sigma\left(0^{+}\right)\right] \hat{\sigma}\left(0^{+}\right) J[\psi(t)]\right\} \\
& +g_{1}[\sigma(t)]\left\{\int_{0}^{t} J[\psi(t)-\psi(\tau)] \frac{\mathrm{d}}{\mathrm{d} \tau}[\hat{\sigma}(\tau)] \mathrm{d} \tau\right\} \\
& +\left\{\sum_{i=1}^{n} D_{i} J\left[\psi(t)-\psi\left(t_{i}\right)\right]\right\}
\end{aligned}
$$

where,

- $\varepsilon(t)$ and $\sigma(t)$ are respectively linear strain and Cauchy stress tensors. $J(t)$ stands for the creep compliance as a function increasing with time and if necessary, as a function of temperature.

- $t$ denotes the actual time and $\tau$ is a variable ranging over an interval representing the time of load history and called elapsed time.

- $g_{1}[\sigma(t)]$ is a stress dependent factor which expresses the non-linear memory of the material. The function $g_{1}$ is equal to 1 at low stress (linear viscoelasticity) while under high stress levels it arrows proportionally to stress. In the case of polymers, a slope generally between $0.04 \mathrm{MPa}^{-1}$ and $0.05 \mathrm{MPa}^{-1}$ characterises the evolution of the function $g_{1}$ (at high stress), Zaoustos [30].

- $\hat{\sigma}(t)=\sigma(t) g_{2}[\sigma(t)], g_{2}[\sigma(t)]$ is known as the hardening stress factor. $g_{2}$ is equal to 1 at low stress (linear viscoelasticity) while under high stress it arrows proportionally to stress. In the case of polymers loaded at high stress, the evolution of the function $g_{2}$ is characterised by a slope between $0.05 \mathrm{MPa}^{-1}$ and $0.06 \mathrm{MPa}^{-1}$, Zaoustos [30].

$$
\psi(t)=\int_{0}^{t} \frac{\mathrm{d} \tau}{a_{\sigma}[\sigma(\tau)]}
$$

is the reduced time (Eq. (1)) and $\tau$ is the elapsed time

- $a_{\sigma}[\sigma(t)]$ is the multiplying factor called stress-time multiplying factor.

- $D_{i}=g_{1}\left[\sigma\left(t_{i}^{+}\right)\right] \hat{\sigma}\left(t_{i}^{+}\right)-g_{1}\left[\sigma\left(t_{i}^{-}\right)\right] \hat{\sigma}\left(t_{i}^{-}\right)$is the possible stress step at time $t=t_{i}, i=1,2, \ldots n . t_{i}^{-}$and $t_{i}^{+}$are times of stress discontinuity, $t_{i}^{-} \leq t_{i}$ and $t_{i}^{+} \geq t_{i}$.

Schapery nonlinear viscoelastic theory is used as a basis to extend the Dynamic Mechanical Tests and Analysis (DMTA) approach to nonlinear characterization, Zaoustos [30]. Hence, the time required to determine the nonlinear parameters is substantially reduced using the DMTA methodology compared to creep and recovery tests. Among the motivation of the Schapery model is that is close to visco-elasticity law. In addition, it is written in a simple form in the case of creep.

Integrating by parts, equation (2) leads to the following expression of Schapery's model:

$\varepsilon(t)=J\left(0^{+}\right) g_{1}[\sigma(t)] \hat{\sigma}(t)+g_{1}[\sigma(t)] \int_{0}^{t} \dot{J}[\psi(t)-\psi(\tau)][\hat{\sigma}(\tau)] \mathrm{d} \tau$

where $\dot{J}$ denotes the derivative of creep function $J(t)$. The first right side term in Equation (4) represents the instantaneous elastic behaviour of the material, and the second term the memory effect. Possible discontinuities of stress are systematically taking into account in Equation (4). We insist on the fact that the original Schapery model is invariant with respect to time and can be used only for non ageing materials. 
Experimental characterization and validation of the Schapery model when, among others, performed on stratofilm (a linear low density polyethylene film), Golden [31]. We should mention that the Schapery model is a special case of Knauss and Emri work [27,28]) which have noted that the actual time scale can be modified by a large number of internal variables (moisture, dilatation, ...) in the same manner as by temperature. These effects are commonly associated with changes in the free volume of material that offers a unifying parameter to describe changes in time scale. Moreover, O'Dowd et al. [3] presented a generalised form of the Schapery model which describes deformation of polymers under both small and large strains. It uses the kinetic theory of rubber elasticity as a starting point for large strain behaviour and generalizes this theory to include the effects of rate dependence.

On the other hand, Straganac et al. [32] have provided a simplification of Schapery's model to predict the elastomer's creep response, in finite deformations, due to a single step load.

Creep function $J$ was obtained from measurements associated with linear viscoelastic response and it can be written by a parametric expression (exponential law, power law, ...).

\subsection{A generalized Schapery model}

Generalizing Knauss and Emri suggestions [27], the reduced time can be expressed in the following differential equation (see Eq. (3)):

$$
\mathrm{d} \psi=\frac{\mathrm{d} t}{a_{\sigma}[\sigma(t)]}
$$

The concept of free volume lied in the fact that temperature, stress solvent concentration, etc. affect the fractional free volume in the material and consequently the mobility of inclusions and the change in fractional free volume due to any one of internal variable is additive. Mobility of various inclusions generates macroscopic damping in material that can be included in time behaviour laws. Let us assume that the reduced time $\mathrm{d} \psi$ is a superposition of actual reduced time $\mathrm{d} \psi_{0}$ and elapsed reduced time $\mathrm{d} \psi_{\mathrm{e}}$ :

$$
\mathrm{d} \psi=\mathrm{d} \psi_{0}+\mathrm{d} \psi_{\mathrm{e}}
$$

These effects can also be associated with changes of two kinds of free volumes in rubber materials:

- the free volume associated at the molecular scale (micro level of matrix) which is generated by $\mathrm{d} \psi_{0}$ defined by Knauss and Emri [27] ;

- the free volume associated at the mesoscopic scale such as discontinuities between separation of phases (meso level of carbon black) which takes into account the matrix's viscoelasticity (rubber). This is generated by $\mathrm{d} \psi_{\mathrm{e}}$.

In the Schapery model, time is then artificially tensed up or expanded by using a shift factor which depends on both stress and time. Instead of Equation (5) we take into account Equation (6) and the shift factor is defined by the following relation:

$$
\psi(t)=\int_{0}^{t} \frac{\mathrm{d} s}{\alpha_{\sigma}[\sigma(s), t-s]}
$$

where $\alpha_{\sigma}(\sigma, t)$ is the generalized time-stress multiplying factor.

$\alpha_{\sigma}(0, t)=$ const., $\forall t$ when stress is null. Looking at the derivative of relation ( 7 ) one can notice, taking into account relationship (6), that actual reduced time $\mathrm{d} \psi_{0}$ and elapsed reduced time $\mathrm{d} \psi_{h}$ are solutions of the following integro-differential equations:

$\mathrm{d} \psi_{0}=\frac{\mathrm{d} t}{\alpha_{\sigma}[\sigma(t), 0]}, \quad \mathrm{d} \psi_{\mathrm{e}}=\left\{\int_{0}^{t} \frac{\partial\left[\frac{1}{\alpha_{\sigma}[\sigma(t), t-s]}\right]}{\partial t} \mathrm{~d} s\right\} \mathrm{d} t$

As in classical Schapery model, it is easy to show the invariance of this generalized model with respect to time in the case of non ageing materials. That means, if we have the stress/strain state $(\sigma(t), \varepsilon(t))$ at time $t$, we will have stress/strain state $(\sigma(t+\tau), \varepsilon(t+\tau))$ at time $(t+\tau)$. When stress is a single step function:

$$
\begin{aligned}
& \sigma(t)=\sigma_{0} \text { if } t>0 \\
& \sigma(t)=0 \text { if } t<0
\end{aligned}
$$

Relations (2), bringing down to the first right term of Equation (7), provide the creep function $J$ represented by the following equation:

$$
\varepsilon\left(\sigma_{0}, t\right)=\sigma_{0} g_{1}\left(\sigma_{0}\right) g_{2}\left(\sigma_{0}\right) J\left[\int_{0}^{t} \frac{\mathrm{d} s}{\alpha_{\sigma}\left(\sigma_{0}, t-s\right)}\right]
$$

Assume that the classical time-stress multiplying factor $a_{\sigma}$ is balanced by a weight function $\frac{1}{1+f(t)}$, then the generalized stress-time multiplying factor is given by:

$$
\alpha_{\sigma}[\sigma(s), t-s]=\frac{a_{\sigma}[\sigma(s)]}{1+f[\sigma(s), t-s)]}
$$

Thus, the reduced time $\psi(\mathrm{t})$, Equation (7), as regards the stress step function $\sigma_{0}$ can be written in following form:

$$
\psi(t)=\frac{t+F\left(\sigma_{0}, t\right)-F\left(\sigma_{0}, 0\right)}{a_{\sigma}\left(\sigma_{0}\right)}
$$

where $F(\sigma, t)$ is the primitive of $f(\sigma, t)\left(\frac{\partial F(\sigma, t)}{\partial t}=f(\sigma, t)\right)$

Finally, when stress is a step function, the creep function (8), taking into account Equation (9), leads to the following form:

$$
\varepsilon\left(\sigma_{0}, t\right)=\sigma_{0} g_{1}\left(\sigma_{0}\right) g_{2}\left(\sigma_{0}\right) J\left[\frac{t+F\left(\sigma_{0}, t\right)-F\left(\sigma_{0}, 0\right)}{a_{\sigma}\left(\sigma_{0}\right)}\right]
$$




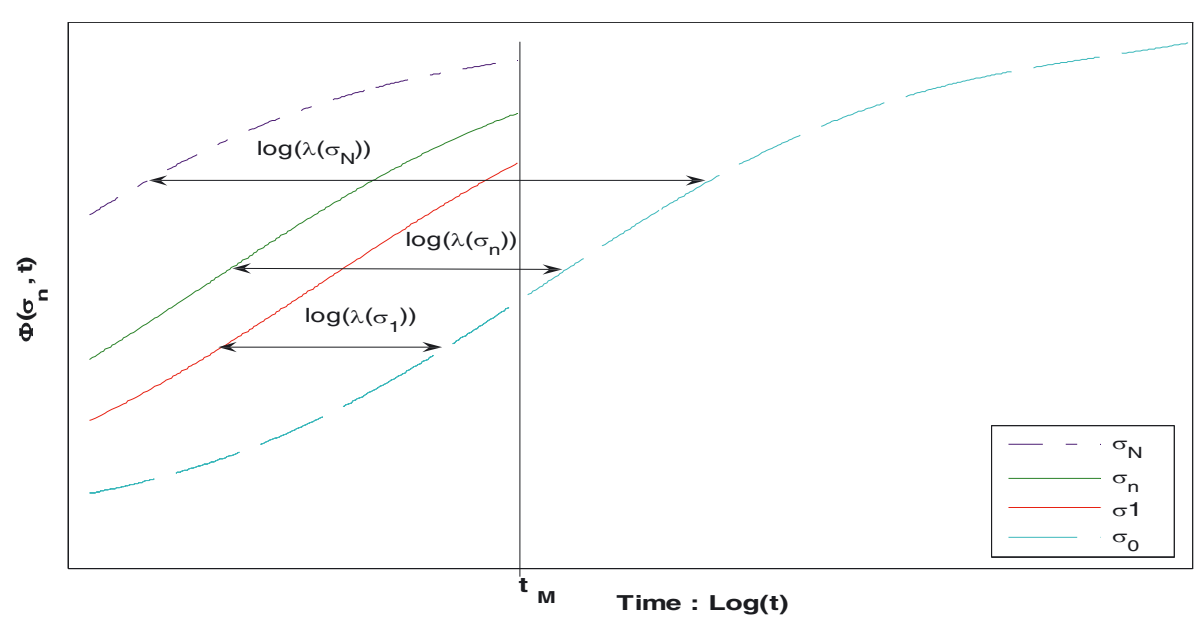

Fig. 1. Scheme of experimental data: reduced creep strain against time (measurements at various preloads $\left(0<t<t_{M}\right)$ ). Classical approach of master curve at $\sigma_{0}$ (lower curve) for longer time periods), $\lambda$ is the constant horizontal shift factor.

\section{Prediction of long term response}

To identify generalized stress-time multiplying factor $\frac{a_{\sigma}[\sigma(s)]}{1+f[\sigma(s), t-s]}$ (Eq. (9)) in the generalized Schapery model, a specific approach has been developed. The idea is that an increase in time is equivalent to an increase in stress. In an analogous context, creep properties measured with a given preload and at a given time may be shown to correspond to the creep properties under another preload and at another time by applying a preload dependent on the generalized shift factor. The shift factor expression is determined by the superposition of measurements with distinct preloads $\sigma_{0}, \sigma_{1}, \sigma_{2, \ldots}, \sigma_{n, \ldots,} \sigma_{N}$. Thus, step function $\sigma$, generates strain according to relation (11) with $F(\sigma, 0)=0$. The objective is to determine the time-stress multiplying factor $a_{\sigma}\left(\sigma_{n}\right)$ and the function $F(\sigma, t)$. Looking at classical Schapery model (Sect. 2.1), weight functions $g_{1}[\sigma(t)]$ and $g_{2}[\sigma(t)]$ can be considered as unit step functions for low stress, Zaoustos and Papanicolaou [30], then Equation (11) becomes:

$$
\varepsilon\left(\sigma_{n}, t\right)=\sigma_{n} J\left[\frac{t+F_{n}(t)}{a_{\sigma}\left(\sigma_{n}\right)}\right] n=0,1,2, \ldots, N
$$

where $F_{n}(t)=F\left(\sigma_{n}, t\right)$.

From creep strain $\varepsilon\left(\sigma_{n}, t\right)$ the reduced creep function $\Phi\left(\sigma_{n}, t\right)=\frac{\varepsilon\left(\sigma_{n}, t\right)}{\sigma_{n}}$ is plotted against actual time at various stress levels, $n=0,1,2, \ldots, N$. Taking into account equation (12) reduced creep strain is given by the following relation:

$$
\Phi\left(\sigma_{n}, t\right)=J\left[\frac{t+F_{n}(t)}{a_{\sigma}\left(\sigma_{n}\right)}\right]
$$

Properties measured under one preload are equivalent to those under a second preload on a compressed or expanded time scale. Scheme of experimental data are plotted in Figure 1.

Using the different classical horizontal shift factors $\lambda($ Eq. (3))

$$
\lambda\left(\sigma_{n}\right)=\frac{a_{\sigma}\left(\sigma_{0}\right)}{a_{\sigma}\left(\sigma_{n}\right)}
$$

between one curve at a reference stress $\sigma_{0}$, and other curves at different stresses $\sigma_{n}$, a master curve of the compliance can be formed for a reference preload.

We used the generalized shift factors between short term creep curves to display (or to complete) a master curve over a longer time period using the classical method (Fig. 1). This master curve describes the behaviour over a broad time range. The effect of a change in preload is equivalent to measurements on a different time scale, that means if $\Phi\left(\sigma_{0}, t_{0}\right)=\Phi\left(\sigma_{n}, t_{n}\right)$ for two preloads $\sigma_{0}$ and $\sigma_{n}$, then $J\left[\frac{t_{0}+F_{0}\left(t_{0}\right)}{a_{\sigma}\left(\sigma_{0}\right)}\right]=J\left[\frac{t_{n}+F_{n}\left(t_{n}\right)}{a_{\sigma}\left(\sigma_{n}\right)}\right]$ for, $n=1,2, \ldots, N$.

Assume that $\sigma_{0}$ is the lower curve (Fig. 1). If $\Phi\left(\sigma_{0}\right.$, $\left.t_{0}\right)=\Phi\left(\sigma_{n}, t_{n}\right)$, then shift times are related by the equation below on a logarithmic scale:

$$
\log \left(t_{0}+F_{0}\left(t_{0}\right)\right)=\log \left(t_{n}+F_{n}\left(t_{n}\right)\right)+\log \left[\lambda\left(\sigma_{n}\right)\right]
$$

where $\lambda\left(\sigma_{n}\right)=\frac{a_{\sigma}\left(\sigma_{0}\right)}{a_{\sigma}\left(\sigma_{n}\right)}$ is the classical constant horizontal shift factor, on a logarithmic scale, between reduced creep strains $\Phi\left(\sigma_{0}, t_{0}\right)$ and $\Phi\left(\sigma_{n}, t_{n}\right)$. The lower curve $\left(\sigma=\sigma_{0}\right)$ is considered, for example, as a master curve. Then $F_{0}\left(t_{0}\right)=0$ and two zones can be graphically detected looking at Figure 2:

- zone 1: $t<t_{\min }$ (Fig. 2): reduced creep strain functions for various loadings are parallel (Fig. 2) and thus the time dependent horizontal shift factor $\zeta\left(\sigma_{n}, t_{n}\right)$ is determined in this range time by the straight line $\mathrm{A}_{1} \mathrm{~B}_{1}$ on a logarithmic scale, (Fig. 2). By choosing $F_{n}=0$ and taking into account relation (15) we obtain the following equation:

$$
A_{1} B_{1}=\log \left(t_{0}\right)-\log \left(t_{n}\right)=\log \left[\zeta\left(\sigma_{n}, t_{n}\right)\right]=\log \left[\lambda\left(\sigma_{n}\right)\right]
$$

Hence, in this area 1, the time dependant horizontal shift factor does not depend upon time and is expressed by the following relation:

$$
\zeta\left(\sigma_{n}, t_{n}\right)=\lambda\left(\sigma_{n}\right)
$$



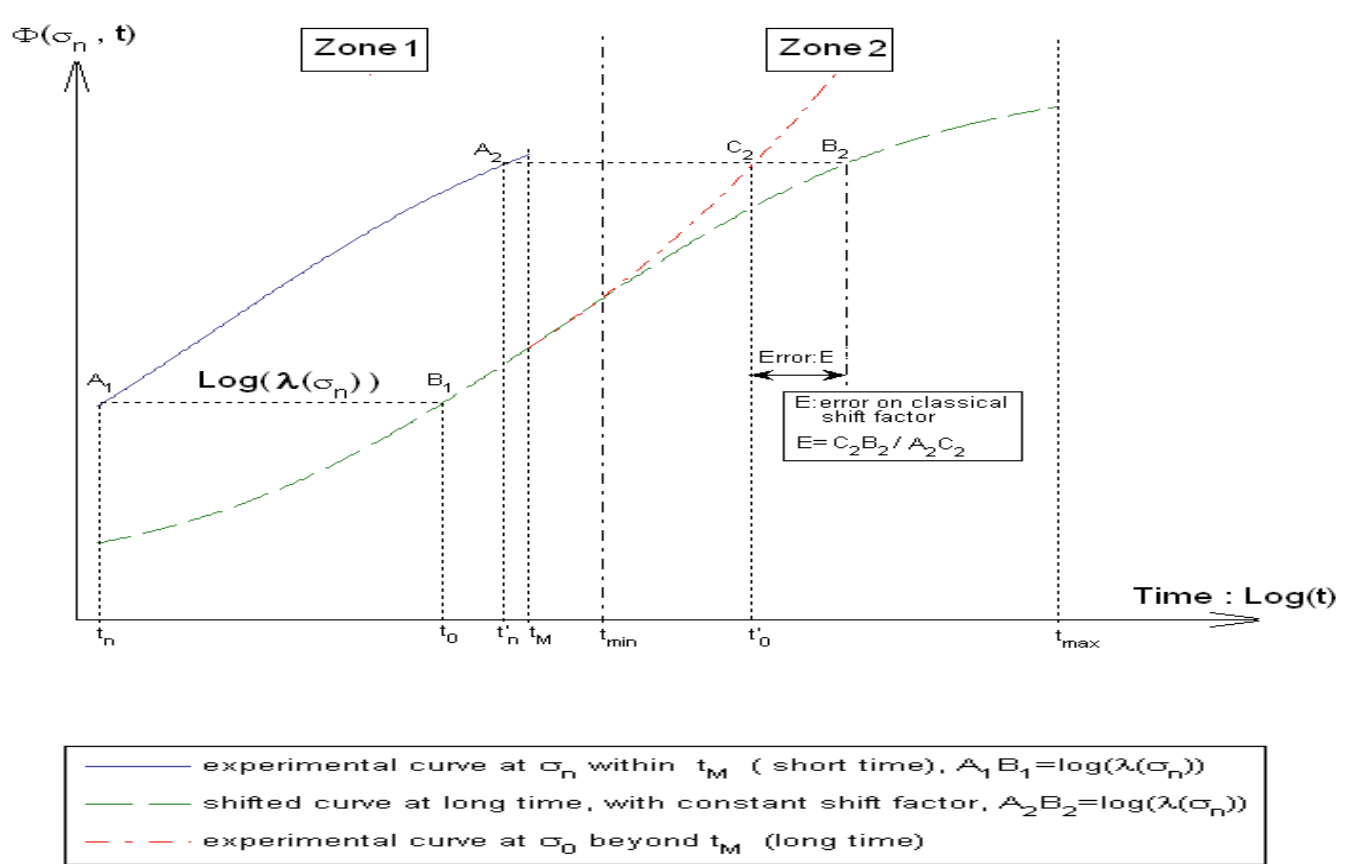

Fig. 2. Scheme of experimental data: reduced creep strain against time (measurements at various preloads $\left.\left(0<t<t_{M}\right)\right)$. New approach of master curve at $\sigma_{0}$ (lower curve) for longer time periods). $\xi$ is the time dependent horizontal shift factor, $\lambda$ is the constant horizontal shift factor.

- zone 2 (Fig. 2): $t_{\min }<t<t_{\max }$. We use the constant horizontal shift factor $\lambda_{n}$ to complete the master curve over a long time period at a reference stress $\sigma_{0}$, (dashed line). A discrepancy can be denoted between shifted curve and experimental curve and thus the time dependent shift factor $\zeta\left(\sigma_{n}, t_{n}^{\prime}\right)$ can be described through the following relation:

$$
A_{2} C_{2}=\log \left(t_{0}^{\prime}\right)-\log \left(t_{n}^{\prime}\right)=\log \left[\zeta\left(\sigma_{n}, t_{n}^{\prime}\right)\right]
$$

Using the time dependant horizontal shift factor, balance Equation (15), and $F_{0}\left(t_{0}\right)=0$ :

$$
\log \left(t_{0}^{\prime}\right)=\log \left(t_{n}^{\prime}+F_{n}\left(t_{n}^{\prime}\right)\right)+\log \left[\lambda\left(\sigma_{n}\right)\right]
$$

Thus, Equation (16) becomes:

$$
\log \left[\zeta\left(\sigma_{n}, t_{n}^{\prime}\right)\right]=\log \left\{\lambda\left(\sigma_{n}\right)\left[1+\frac{\left.F_{n}\left(t_{n}^{\prime}\right)\right)}{t_{n}^{\prime}}\right]\right\}
$$

From which the time dependent horizontal shift factor $\zeta\left(\sigma_{n}, t_{n}^{\prime}\right)$ is defined at time $t_{n}^{\prime}$ by the following relation:

$$
\zeta\left(\sigma_{n}, t_{n}^{\prime}\right)=\lambda\left(\sigma_{n}\right)\left[1+\frac{F_{n}\left(t_{n}^{\prime}\right)}{t_{n}^{\prime}}\right]
$$

Creep master curves, however, do not enjoy the same translation factor for all time ranges. A discrepancy can be denoted at long time between shifted curve with classical (constant) shift factor and the experimental curve. We mention that the error $E$ between these two curves can be characterized by the quotient $E=C_{2} B_{2} / A_{2} C_{2}$.

Hence, the shift factor is not only a function of stress but may be also a function of time and thus confirms the generalized Schapery model.

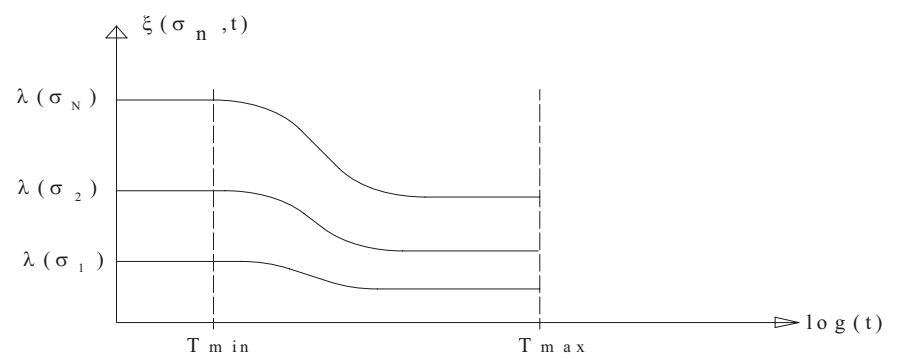

Fig. 3. General time dependent horizontal shift factor as function of time.

In short, the generalized Shapery method used to predict long time behaviour of materials is summarized in a pair of Equations (18) and (19) providing the time dependent shift factor and a wide range of experimental curves at various stresses $\sigma_{n}$ (Fig. 3) which allow the function $F(t)$ and the constant $K_{n} n=1,2, \ldots, N$ to be updated.

$$
t<t_{\min }: \zeta\left(\sigma_{n}, t\right)=\lambda\left(\sigma_{n}\right)
$$

$$
t_{\min }<t<t_{\max }: \zeta\left(\sigma_{n}, t\right)=\lambda\left(\sigma_{n}\right)\left[1+\frac{\left.F_{n}(t)\right)}{t}\right]
$$

\section{Experiment and analysis}

In this section we present an experimental identification of viscoelastic response using creep tests. Figures 4 and 5 describe the creep test apparatus which is equiped 


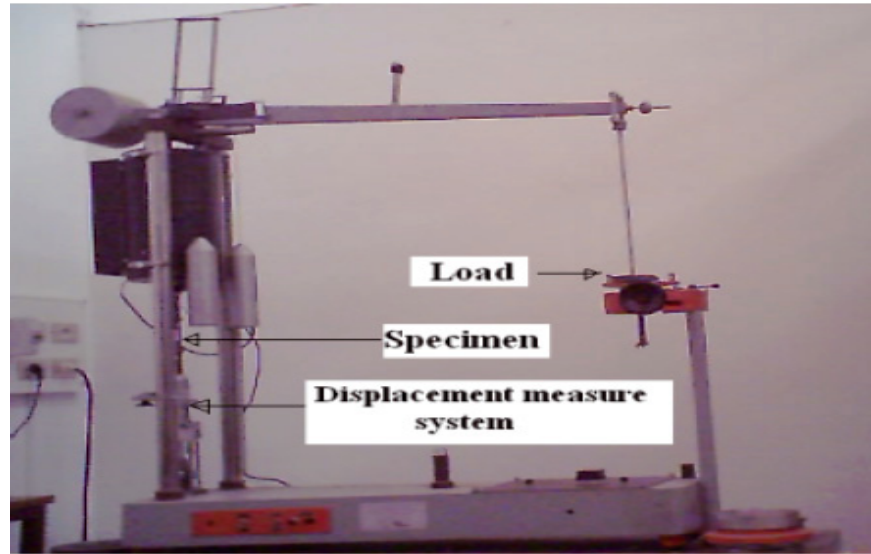

Fig. 4. Experimental apparatus for creep test.

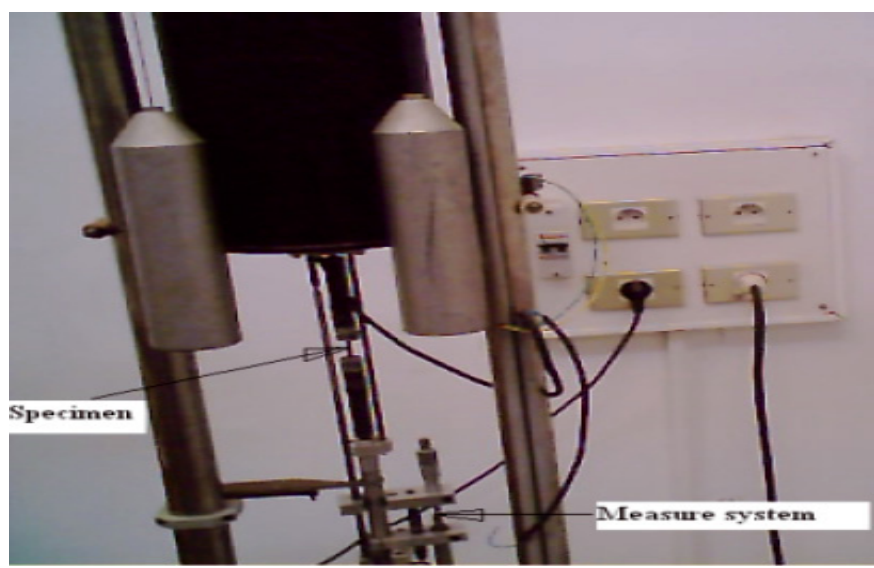

Fig. 5. Displacement measurement system.

with an electronic displacement measuring system (transducer + data airing card + software).

The normalized specimen, Figure $6 \mathrm{a}$, which is made of a synthetic vulcanised elastomer is used to conduct creep tests at various stress levels. Its glass transition temperature is approximately $248 \mathrm{~K}$. This material is used in manufacturing classical multilayer plate incorporating viscohyperelastic materials. This latter is confined between the stiff layers and worked as a damping layer. Four parts make up the test device (Fig. 6b)

- 1 and 4: fastening jaws;

-2 : bridle fixed on jaw;

- 3: rod guide;

- 5: sole plate fixed on specimen.

Creep tests results at different preloads are presented in Figure 7. All experimental results are obtained for $160 \mathrm{~h}$ at various stresses from $0.5 \mathrm{MPa}$ to $2.5 \mathrm{MPa}$. These curves show the instantaneous elastic deformation at the beginning of the test. These elastic deformations increase with the preload. The strain rate depends on time and preload.

To better observe relations between creep curves using a shift factor, these curves are transformed into a semilogarithmic scale representation (Fig. 8). The compliance modulus presented in Figure 8 is the reduced creep strain (Eq. (13)).

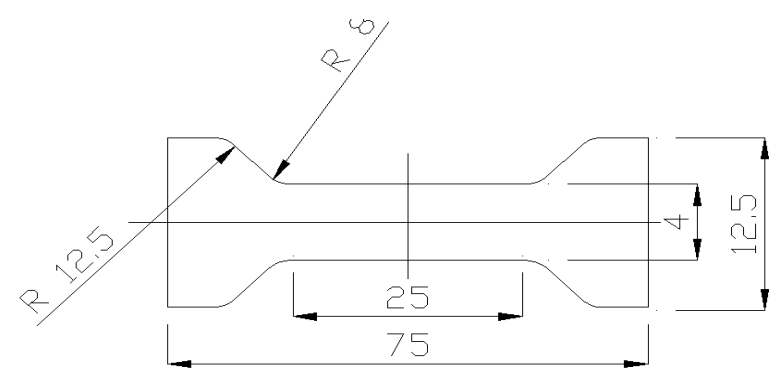

(a)

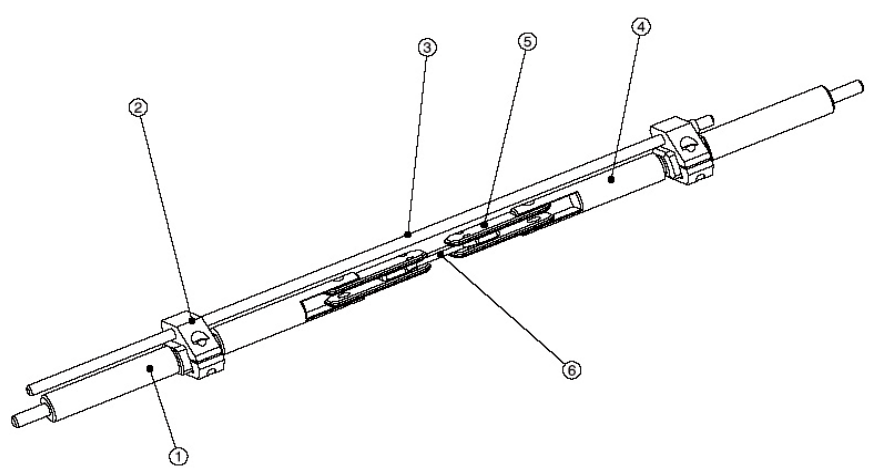

(b)

Fig. 6. Scheme of specimen and test device. (a) Normalized Specimen "Haltere - H2" for creep tests (dimension in $\mathrm{mm}$ ). Thickness $=2.5 \mathrm{~mm}$; useful length $=20 \mathrm{~mm}$. (b) Setting of specimen.

A long term creep test is performed at a reference preload of $2.5 \mathrm{MPa}$ and with duration of about 115 days (Fig. 9). This curve is considered as a master curve and can be plotted using time-stress superimposition by using a convenient shift factor expression with short term creep curves. Thus, this approach enables us to complete a master curve over a long time period. This master curve displays the material behaviour over a wide time range. In Figure 9, a discrepancy can be denoted at long time between shifted curve with classical constant horizontal shift factor $\lambda\left(\sigma_{n}\right)$ (classical theory Eq. (14)) and the experimental long term curve at a preload of $2.5 \mathrm{MPa}$. We characterise this discrepancy by defining the error $E$ as follows

$$
E=\frac{C_{2} B_{2}}{A_{2} C_{2}}
$$

$\left(C_{2} B_{2}\right)$ characterizes the difference between the long term experimental curve and the curve obtained from short time tests using classical shift factor $\lambda\left(\sigma_{n}\right) .\left(A_{2} C_{2}\right)$ characterizes the shifted time between the long term experimental curve and the short term one.

We mention that the error $E$ between these two curves is unacceptable and higher than $1600 \%$ (Fig. 9). Hence, we developed an extended expression for the shift factor which takes into account time (Eq. (19)).

Figure 10 shows the time shift factor evolution between a reference curve at $2.5 \mathrm{MPa}$ and curves at $0.5 \mathrm{MPa}$, $1 \mathrm{MPa}$ and 1.5 MPa. We observe a constant shift factor at short time intervals. However the shift factor 


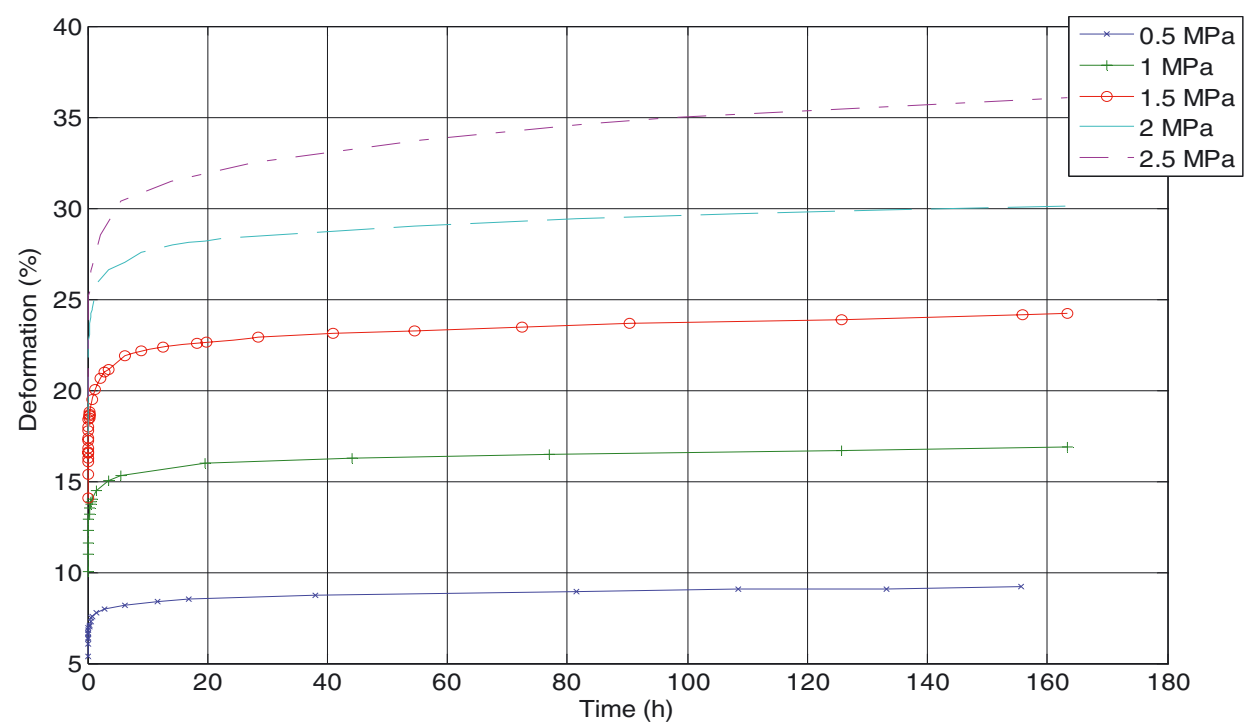

Fig. 7. Creep tests of an elastomer at different preloads.

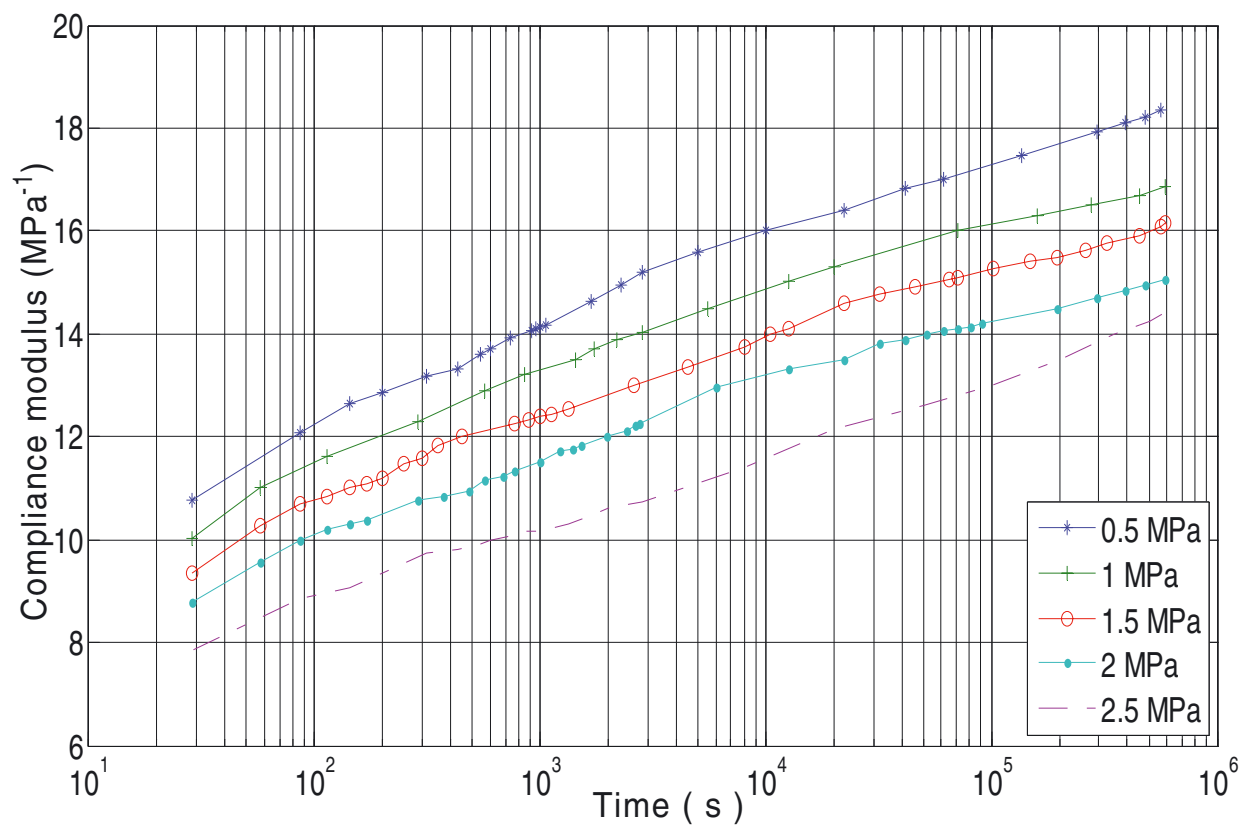

Fig. 8. Creep tests of an elastomer at different preloads.

decreases with time over long periods. We should note that the shift factor is considered after the instantaneous response in the creep curves. To present a quasi-constant shift factor at short and very long time periods with a transition domain which is illustrated in Figure 10, we choose relation (21) for fitting the shift factor according to Equation (19).

$$
\zeta\left(\sigma_{n}, t\right)=\lambda\left(\sigma_{n}\right)\left[1-\frac{2}{\pi} \arctan \left(\mathrm{e}^{a \frac{t-b}{t}}\right)\right]
$$

In the above relation, $F_{n}(t)$ is taken to be:

$$
F_{n}(t)=F\left(\sigma_{n}, t\right)=-\frac{2 t}{\pi} \arctan \left(\mathrm{e}^{a \frac{t-b}{t}}\right)
$$

$a$, and $b$ are material's constants independent of stress levels which are given through stress step tests. Function $F(\sigma, t)$ is mapped out with a set of curves fitting time dependent horizontal shift factors as function of time at various stress steps (see Fig. 10).

\section{Conclusions}

In this work we have first outlined the progress in modelling nonlinear elastomer behaviour. We have reviewed the basic approaches. We stress nonlinear viscoelasticity in terms of behavioral non linearity. A fundamental approach based on the Schapery model, involving a functional approach is described. Modelling based on 


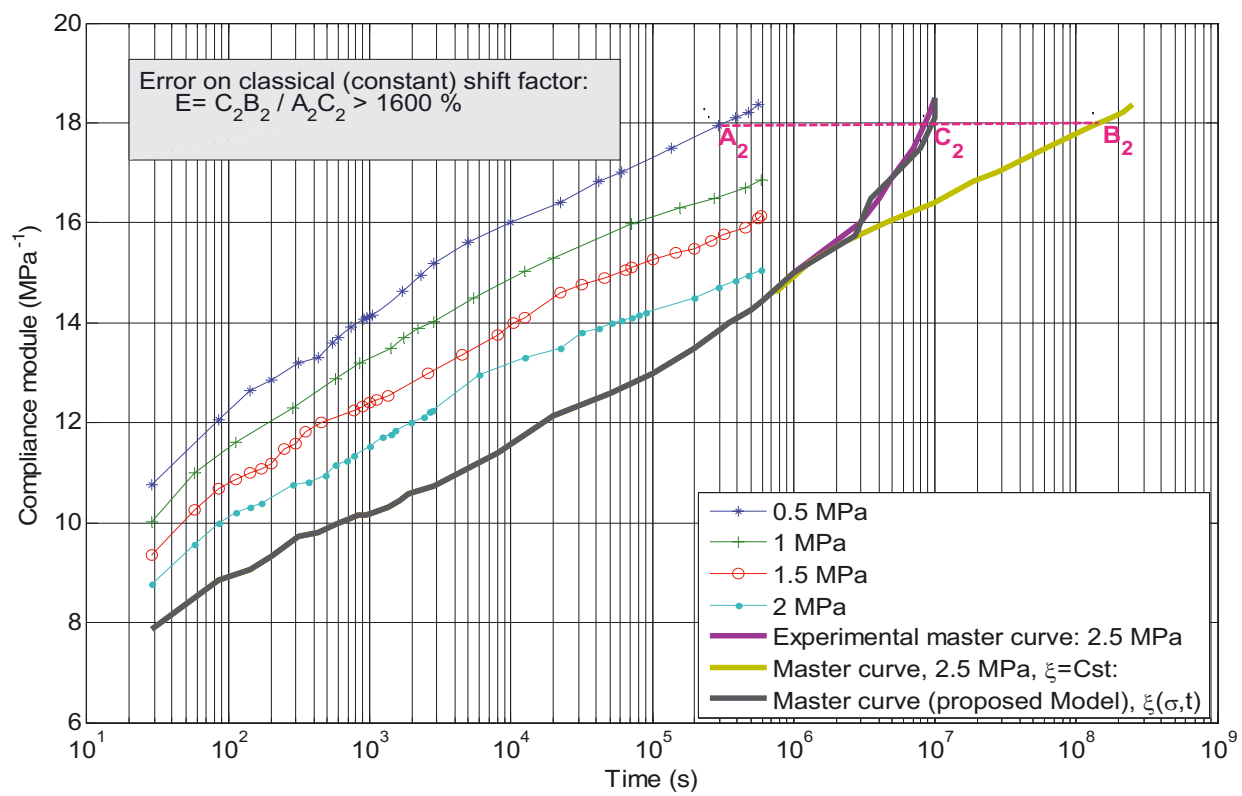

Fig. 9. Creep master curve at stress $=2.5 \mathrm{MPa} .\left(6 \times 10^{5} \mathrm{~s}=7\right.$ days; $10^{7} \mathrm{~s} \approx 115$ days $)$.

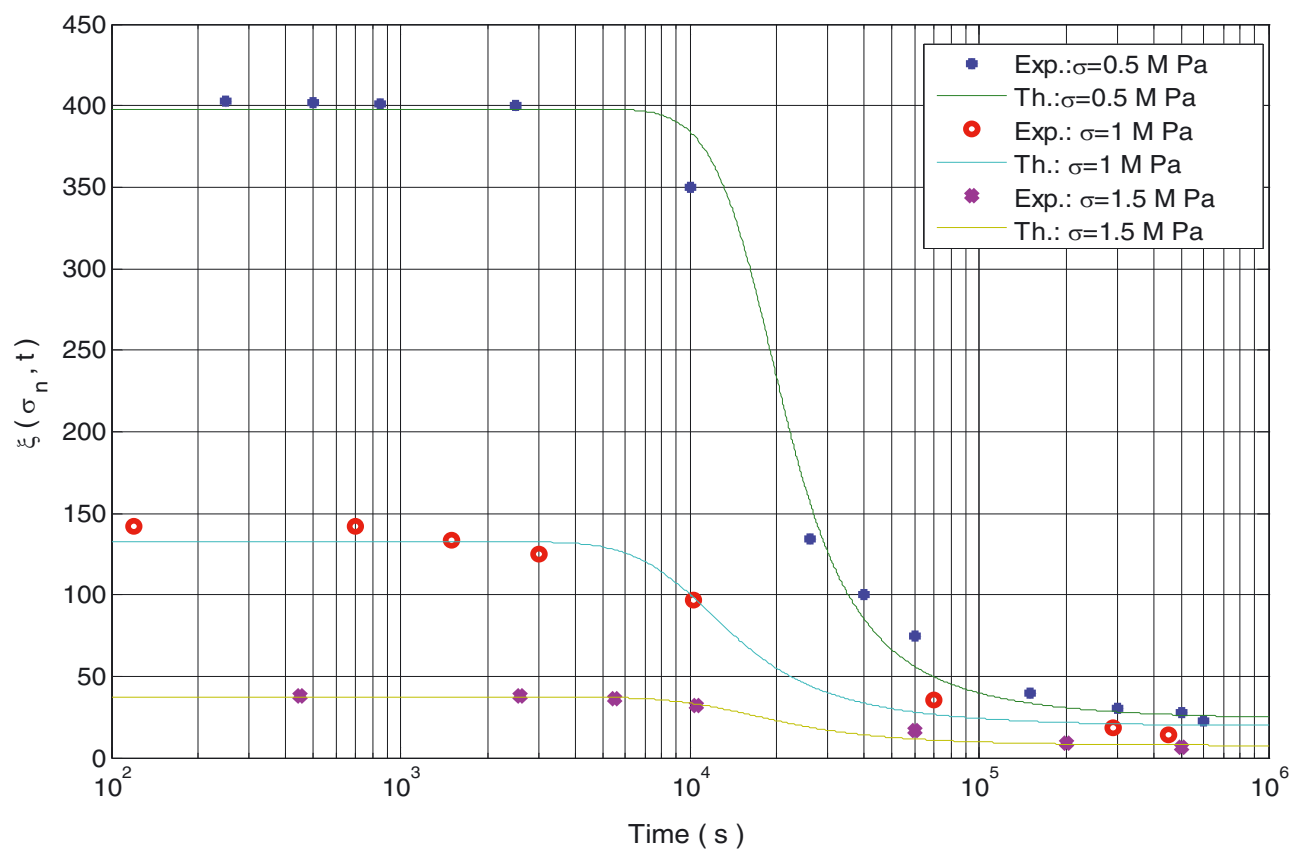

Fig. 10. Time dependant horizontal shift factor fitting for a reference creep curve at $2.5 \mathrm{MPa}$ and a creep curve at $0.5 \mathrm{MPa}$, $1 \mathrm{MPa}$ and $1.5 \mathrm{MPa}(a=1.61 ; b=20970.6) . \sigma=0.5 \mathrm{MPa}: \lambda=397.9 ; \sigma=1 \mathrm{MPa}: \lambda=132.4 ; \sigma=1.5 \mathrm{MPa}: \lambda=37.22$.

this method is intuitive and easier as regards experimental identification. In addition, most of the models so far developed have relied on the functional approach. In this context, we propose an expanded form of the simplified Schapery model to better characterise the creep behaviour for an elastomer. In general, no single "best model" exists. However, various models are valid for particular materials and in specific circumstances.

A characterisation of the non linear visco-elastic behaviour of an elastomer is presented upon creep tests conducted in the short term as in the long term at different preloads. We have checked by means of the experiments that creep functions at various constraints are bound by a shift factor which usually depends on the constraint and unusually of time. Hence, a decrease in the shift factor's variation with time over long period was observed.

Thereafter, an original development generalising modelling the nonlinear viscoelastic behaviour of elastomers is developed. Accordingly, our work is based on a generalization of Schapery model involving the presence in material of two free volumes respectively associated to 
micro level (molecular scale) and meso-level (carbon black inclusions). By developing a time-stress equivalence approach, it is possible to predict the viscoelastic response for material in service over long periods using measurements obtained from short term creep tests.

We could calculate the creep behaviour for 115-day intervals using creep tests for 7 days. This approach has required the development of an original convenient expression for a shift factor which is a function of both stress and time. The prospect of developing this approach for longer time periods and in three-dimensional models will be of great utility for dimensioning complex structures.

\section{References}

[1] P. Saad, Modélisation du comportement viscoélastique non-linéaire des élastomères autour d'une précharge, Mécanique \& Industries 4 (2003) 133-142

[2] A. Amin, M. Alam, Y. OKui, An improved hyperelastic relation in modeling viscoelasticity response of natural and high damping rubbers in compression, experiments, parameter identification and numerical verification, Mechanics of Materials 34 (2002) 75-95

[3] N.P. 0'Dowd, W.G. Knauss, Time dependent large principal deformation of polymers, J. Mech. Phys. Solids 43 (1995) 771-792

[4] G. Lianis, Constitutive equations for viscoelastic solids under finite deformation, J. Appl. Mech. 32 (1965) 623629

[5] K. Hausler, M.B. Sayir, Nonlinear viscoelastic response of carbon black renforced rubber derived from moderately large deformation in torsion, J. Mech. Phys. Solids 43 (1995) 295-318

[6] H. Gacem, Comportement visco-hyperélastique des élastomères, Viscoélasticié non-linéaire, Application aux Multicouches, Thèse de doctorat, Université Pierre \& Marie Curie, Paris 6, décembre 2007

[7] R.A. Schapery, On a time dependence of viscoelastic variational solutions, Q. Appl. Math. 22 (1964) 207-215

[8] R.A. Schapery, Application of thermodynamics to thermomechanical, fracture and birefringent in viscoelastic phenomena, Q. Appl. Math. 22 (1964) 207

[9] R.A. Schapery, Effect of cyclic loading on the temperature in viscoelastic media with variable properties, AIAA J. 2 (1964) 827-835

[10] R.A. Schapery, Thermomechanical behavior of viscoelastic media with variable properties subjected to cyclic loading, J. Appl. Mech. Trans. ASME Series E 87 (1965) 611-619

[11] R.A. Schapery, On viscoelastic deformation and failure behavior of composite materials with trbuted flaws, in advanced in Aerospace Structures and Materials, S.S. Wang \& WJ. Reir (ed.), New York, ASME 1 (1981) 520

[12] R.A. Schapery, Analysis of damage growth in particulate composites using work potential, Co Engrg. 1 (1991) 167182

[13] R.A. Schapery, Nonlinear viscoelastic and viscoplastic constitutive-equation with growing damage, Internat. J. Fracture 97 (1999) 33-66
[14] R.M. Hinterhoelzl, R.A. Schapery, Fem implementation of a three-dimensional viscoelastic constitutive model for particulate composite with damage growth, Mechanics of time dependent Materials 8 (2004) 65-94

[15] R.A. Schapery, An engineering theory of nonlinear viscoelasticity with applications, Int. J. Solids Structures 2 (1966) 407-426

[16] R.A. Schapery, A thermodynamic constitutive theory and its application to various nonlinear materials, Int. J. Solids Structures 2 (1966) 407-425

[17] R.A. Schapery, Nonlinear Viscoelastic characterization and stress analysis of solid propellants, Review Article, Solid Rocket Structural Integrity Abstracts, College of Engineering of Utah, Vol. 3, Series E, September, 1966

[18] R.A. Schapery, Stress analysis of viscoelastic composite materials, J. Composite Mat. 1 (1967) 228-267

[19] J.D. Ferry, Viscoelastic properties of polymers, John Wiley and Sons, 3rd edition, New York, 1980

[20] M.L. Morland, E.H. Lee, Stress analysis for linear viscoelactic materials with temperature variation, Trans. Soc. Rheology 4 (1960) 233-263

[21] E.H. Lee, T.G. Roberts, On the generation of residual stress in thermoviscoelastic bodies, J. Appl. Mech. 65 (1965)

[22] R.A. Schapery, Correspondence Principles and a generalized J Integral for large deformation fracture analysis of viscoelastic media, Int. J. Fracture 25 (1984) 195-223

[23] F. Sidoroff, Variables internes en visco-elasticité, variables internes scalaires et tensorielles, J. Mécanique 14 (1975) 545-566

[24] L. Onsager, Reciprocal relations in irreversible processes, Phys. Rev. (I) 37 (1931) 405-426

[25] L. Onsager, Reciprocal relations in irreversible processes, II. Phys Rev (II) 38 (1931) 2265-2279

[26] M. Lévesque, Modélisation du comportement mécanique des matériaux composites viscoélastiques non linéaires par une approche d'homogénéisation, Thèse de doctorat, ENSAM Paris, Décembre 2004

[27] W.G. Knauss, I.J. Emri, Non-linear viscoelasticity based on free volume consideration, Computers \& Structures 13 (1981) 123-128

[28] W.G. Knauss, I.J. Emri, Volume change and the nonlinearly thermo-viscoelastic constitution of polymers, Poly. Eng. Sci., A. Yee (ed.) 27 (1987) 86-100

[29] A.K. Doolittle, Studies on Newtonian flow II; The dependence of the viscosity of liquid on free space, J. Appl. Phys. 22 (1950) 1471-1475

[30] S.P. Zaoutsos, G.C. Papanicoaou, Study of the effect of fibre orientation on the non-linear viscoelastic behaviour of continuous fibre polymer composites. Recent developments in durability analysis of composite systems, Cardon, Fukuda, Reifsnider \& Verchery (ed.), 2000, Belkema, Rotterdam, pp. 375-379

[31] H.J. Golden, An approach to characterize nonlinear viscoelastic material behaviour using dynamic mechanical tests and analyses, J. Appl. Mech. 66 (1999) 872-878

[32] T.W. Straganac, H.J. Golden, Prediction of nonlinear viscoelastic behaviour using hybrid approach, Int. J. Solid Structures 33 (1996) 4651-4570 